\title{
Pathogenesis and clinical perspectives of extraintestinal manifestations in inflammatory bowel diseases
}

\author{
Jung Min Kim, ${ }^{1}$ Jae Hee Cheon ${ }^{1,2,3}$ \\ ${ }^{1}$ Department of Internal Medicine and Institute of Gastroenterology, Yonsei University College of Medicine, Seoul; ${ }^{2}$ Avison Biomedical Research \\ Center, Severance Hospital, Seoul; ${ }^{3}$ Affiliate Faculty, Pohang University of Science and Technology (POSTECH), Pohang, Korea
}

A considerable number of patients with inflammatory bowel disease (IBD) experience extraintestinal manifestations (EIMs), which can present either before or after IBD diagnosis. Unraveling the pathogenic pathways of EIMs in IBD is challenging because of the lack of reliable criteria for diagnosis and difficulty in distinguishing EIMs from external pathologies caused by drugs or other etiologies. Optimizing treatment can also be difficult. Early diagnosis and management of EIM revolve around multidisciplinary teams, and they should have the resources necessary to make and implement appropriate decisions. In addition, specialists of the affected organs should be trained in IBD treatment. Furthermore, patient awareness regarding the extraintestinal symptoms of IBD is of paramount importance for improving patient understanding of disease and health outcomes. Herein, we review the pathogenesis and clinical perspectives of EIMs in IBD. (Intest Res 2020;18:249-264)

Key Words: Inflammatory bowel diseases; Neuromuscular manifestations; Skin manifestations; Eye manifestations

\section{INTRODUCTION}

Inflammatory bowel disease (IBD), including CD and UC, is a chronic, intestinal inflammatory disease that is often associated with the development of various extraintestinal symptoms. Extraintestinal symptoms can be divided into 2 groups: extraintestinal manifestations (EIMs) and extraintestinal complications. EIMs are defined in patients with IBD when an inflammatory pathology is located outside the intestine, the pathogenesis of which depends on the extension/translocation of immune pathways from the intestine, is an independent inflammatory event sustained by IBD, or shares a common environmental or genetic predisposition with IBD. ${ }^{1}$ EIMs can be divided into 3 categories: EIMs directly related to bowel disease activity, including episcleritis, erythema nodosum (EN),

Received October 11, 2019. Revised November 25, 2019.

Accepted March 6, 2020

Correspondence to Jae Hee Cheon, Department of Internal Medicine and

Institute of Gastroenterology, Yonsei University College of Medicine, 50-1

Yonsei-ro, Seodaemun-gu, Seoul 03722, Korea. Tel: +82-2-2228-1990, Fax:

+82-2-393-6884, E-mail: geniushee@yuhs.ac oral aphthous ulcers, and pauciarthritis; those not related to bowel disease activity but simply reflecting the patient's susceptibility to related autoimmune disorders, including ankylosing spondylitis (AS) and uveitis; and those with an unclear relationship to bowel disease activity, including pyoderma gangrenosum (PG) and primary sclerosing cholangitis (PSC). Extraintestinal complications are initiated by the disease itself and consequently include micronutrient deficiency, osteoporosis, peripheral neuropathy, nephrolithiasis, and IBD drug-related side effects. ${ }^{2}$ EIMs or extraintestinal complications occur in various organs and significantly affect the quality of life of patients with IBD (Table 1). Physicians who treat IBD need to keep track of the progress and treatment of these EIMs or extraintestinal complications of the disease through multidisciplinary approaches. Herein, we will focus on and review the pathogenesis and clinical perspectives of EIMs in IBD.

\section{WHY ARE EIMs IMPORTANT IN IBD?}

The prevalence of EIMs is strongly influenced by the age at di- 
Table 1. Extraintestinal Manifestations and Complications Associated with IBD

\begin{tabular}{|c|c|c|c|c|}
\hline \multirow[b]{2}{*}{ System } & \multicolumn{3}{|c|}{ Extraintestinal manifestations (prevalence) } & \multirow{2}{*}{$\begin{array}{l}\text { Complications of IBD } \\
\text { IBD or its treatment }\end{array}$} \\
\hline & $\begin{array}{l}\text { Directly related to } \\
\text { bowel disease activity }\end{array}$ & $\begin{array}{c}\text { Not related to } \\
\text { bowel disease activity }\end{array}$ & $\begin{array}{l}\text { Unclear relationship to } \\
\text { bowel disease activity }\end{array}$ & \\
\hline Joints/bone & $\begin{array}{l}\text { Type } 1 \text { peripheral arthritis } \\
\text { Pauciarthritis }\end{array}$ & $\begin{array}{l}\text { Type } 2 \text { peripheral arthritis } \\
\text { Ankylosing spondylitis } \\
(3 \%-20 \%) \\
\text { Axial arthritis (3\%-25\%) }\end{array}$ & & $\begin{array}{l}\text { Metabolic bone disease } \\
\text { Osteoporosis/osteopenia }\end{array}$ \\
\hline Skin & $\begin{array}{l}\text { Erythema nodosum } \\
(1 \%-15 \%)\end{array}$ & - & Pyoderma gangrenosum (1\%-12\%) & $\begin{array}{l}\text { Drug-induced skin disease (anti- } \\
\text { TNF-induced psoriasis, DILE) } \\
\text { Drug hypersensitivity } \\
\text { Drug-induced skin cancer }\end{array}$ \\
\hline Eye & Episcleritis (<1\%) & Uveitis (3\%-11\%) & - & Drug-induced cataracts \\
\hline $\begin{array}{l}\text { Hepato- } \\
\text { pancreato- } \\
\text { biliary }\end{array}$ & - & - & $\begin{array}{l}\text { Primary sclerosing cholangitis }(0.2 \%-7.5 \%) \\
\text { Nonalcoholic fatty liver disease }(8 \%-59 \%) \\
\text { Autoimmune hepatitis }(0.6 \%-1.6 \%) \\
\text { Granulomatous hepatitis }(<1 \%)\end{array}$ & $\begin{array}{l}\text { Drug-induced liver injury } \\
\text { Drug-induced pancreatitis } \\
\text { GB stones } \\
\text { Portal vein thrombosis }\end{array}$ \\
\hline Coagulopathy & - & - & - & Venous thromboembolism \\
\hline
\end{tabular}

DILE, drug-induced lupus erythematosus; GB, gallbladder.

agnosis and $\operatorname{sex}^{3,4}$ and is reported to be $23.0 \%$ in CD and $24.4 \%$ in UC, according to data from the National Health Insurance System of Korea. ${ }^{3}$ Up to $50 \%$ of patients with IBD experience at least one EIMs, which can develop even before the diagnosis of IBD. ${ }^{5}$ However, these results of EIMs differ greatly among study methods and research designs.

Abdominal pain is a common symptom related to IBD, and pain is the principal symptom in IBD. ${ }^{6}$ Pain is a longstanding problem and can also be seen in EIMs. ${ }^{7}$ During disease flares, pain is present in $50 \%-70 \%$ of patients with IBD, and the health-related quality of life (HRQOL) of these patients was reported to be significantly lower in patients suffering from pain than in those without pain. ${ }^{6,7}$

As such, EIMs are a common problem and may significantly impact the quality of life of patients with IBD, requiring specific treatments depending on the affected organs. Therefore, successful management of EIMs is essential for improving the quality of life of patients with IBD.

\section{PATHOGENESIS OF EIMs}

The pathogenic mechanisms of EIMs have yet to be clearly defined. Defining the pathophysiology of an EIM requires significant research and consensus due to the lack of consistent criteria for diagnosing an EIM and difficulty of distinguishing an EIM from extraintestinal complications or a secondary EIM caused by drugs. EIMs are considered to be the result of an antigen-specific immune response from the intestine to an extraintestinal site or an independent inflammatory event that is initiated or persisting from covalent genetic or environmental risk factors in the host, or from IBD (Fig. 1).

\section{Dysbiosis of Gut Microbiota and Disrupted Gut Barrier in Intestinal Sites}

Short chain fatty acids (SCFAs) enhance intestinal health, creating conditions favorable for resistance to pathogenic bacteria and preventing colitis. SCFAs have been found at significantly lower levels in IBD patients with psoriatic arthritis (PsA). ${ }^{8}$ IBD patients with PsA had a relative decrease in the abundance of Coprococcus and Ruminococcus species. Ruminococcus is a mucin-degrading bacterium that is especially important for maintaining intestinal homeostasis through SCFA production. The receptor activator of nuclear factor kappa- $\beta$ ligand (RANKL)/ osteoprotegerin (OPG) system may be a regulator of intestinal microbiomes, and imbalances through the breakage of RANKL in the gut lumen of patients with PsA can promote dissociation of inflammation to a distal site, such as a joint. ${ }^{8}$

IBD patients with spondyloarthritis (SpA) have an in- 


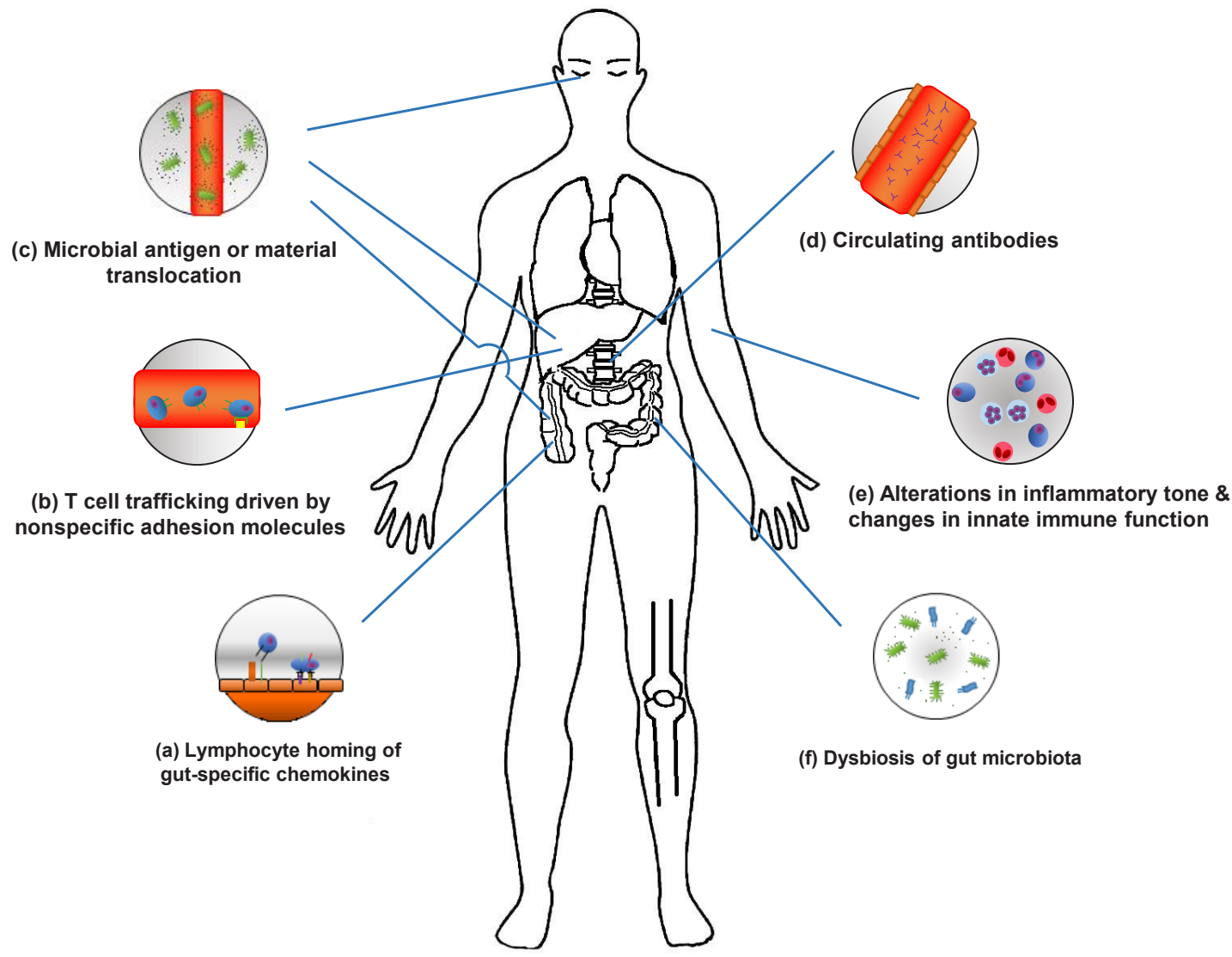

Fig. 1. Mechanisms of extraintestinal manifestations (EIMs). (a) Activation and expansion of effector memory $T$ cells can lead to induction of mucosal vascular addressin cell adhesion molecule 1 and C-C motif chemokine ligand 25 (CCL25) expression in the liver. (b) Intercellular adhesion molecule-1, vascular adhesion protein-1, and vascular cell adhesion molecule 1 are associated with $\mathrm{T}$ cell trafficking. (c) Microbial antigens and materials translocate to extraintestinal sites and drive ElMs. (d) Circulating antibodies can provoke an autoimmune response and peptide(s) expressing common epitopes shared by colon and extra-colonic organs. (e) Neutrophil priming and alteration of circulating monocytes and macrophages can induce intestinal inflammation and are associated with innate immune responses. (f) Gut dysbiosis drives non-intestinal inflammation and increases mucosal permeability and lipopolysaccharides.

creased abundance of the genus Dialister, which is positively correlated with disease activity. ${ }^{9}$ In addition, microbial activation in CD with SpA are associated with interleukin-23 (IL-23) dependent inflammation. ${ }^{10}$ CD-SpA-derived Escherichia coli induced Thelper 17 (Th17) cells polarization and made exacerbation of colitis or inflammatory arthritis.

Gut dysbiosis in IBD patients with AS induces differentiation and activation of group 3 congenital lymphocytes (ILC3s). ${ }^{11}$ ILC3s are IL-23-responsive immune cells that play a pro-inflammatory role in mediating a protective response against extracellular bacterial infections. ILC3s have been identified in the peripheral blood, synovial fluid, and bone marrow tissues of patients with AS, most of which express intestinal homing integrin $\alpha 4 \beta 7$. Mucosal vascular addressin cell adhesion molecule 1 (MAdCAM-1) was highly represented in the bone marrow and AS patients' intestinal high endothelial venules. In addition, MAdCAM-1 plays a central role in attracting ILC3s at the inflammation site of active AS and recirculating ILC3s between the gut and inflamed spines. ${ }^{12}$ In patients with IBD, intestinal effector T cells target gut microbial antigens. Transporting these antigens to the liver through the portal circulation allows the recruitment of mucosal effector $\alpha 4 \beta 7$ positive T cells through $\alpha 4 \beta 7$ MAdCAM-1 interactions.

Patients with PSC showed gut microbial differentiations from those with UC without liver diseases. ${ }^{13}$ In a Gnotobiotic mice model with PSC/UC, Klebsiella pneumoniae, Proteus mirabilis and Enterococcus gallinarum were identified from the mesenteric lymph nodes of PSC/UC Gnotobiotic mice. ${ }^{14}$ Disrupted gut barrier by K. pneumonia is associated with bacterial translocation and Th17 priming to the liver. These bacteria 
can lead the progression of hepatobiliary tract disease through the Th17 response in the colon and liver.

Alterations in the composition and metabolic products of the microbiota can affect the production of innate immune cells in patients with IBD. ${ }^{15,16}$ There were significant differences in the composition of microbiome between HLA-B27+ and HLA-B27- siblings as well as healthy controls, indicating that genetic background can affect the composition and abundance of microbiomes.

Faecalibacterium prausnitzii-specific cells (DP8 $\alpha$ TREG clones), found only in the chemokine receptor $6(\mathrm{CCR} 6)^{+}$and in the C-X-C motif chemokine receptor 6 (CXCR6) fractions of CD4CD8 $\alpha$ (DP8 $\alpha)$ cells, were significantly decreased in the blood of patients with IBD. ${ }^{17}$ These gut recirculating markers are expressed in the DP8 cells of the large intestine and remain expressed in F. prausnitzii-reactive DP8 $\alpha$ cells in the blood, allowing them to move to a specific intestinal position to perform their functions. These characteristics can be conveyed by the joint expression of CCR6 and CXCR6; the expression of CCR6 and CXCR6 also implies homing to other mucosal locations.

\section{Immune Responses in Extraintestinal Sites}

Abnormal lymphocyte homing in IBD can explain why EIMs are associated with site specific distributions. ${ }^{18}$ The $\alpha 4 \beta 7$ integrin is a lymphocyte receptor for the MAdCAM-1. The interaction between $\alpha 4 \beta 7$ and MAdCAM-1 plays an important role in regulating lymphocyte homing to mucosal sites. ${ }^{19}$ Expression of MAdCAM-1 is confined to the intestinal tissue, allowing gut tropic T cells expressing $\alpha 4 \beta 7$ integrin to selectively traffic with the intestinal mucosa. Additional tropism for the small bowel comes from C-C motif chemokine ligand 25 (CCL25) which attracts lymphocytes expressing the CCR9 receptor. T cell migration to the small bowel requires integrin $\alpha 4 \beta 7^{19}$ and CCR9. ${ }^{20}$ Interestingly, homing to the colon requires $\alpha 4 \beta 7$ or $\alpha 4 \beta 1$ but not CCR9. ${ }^{21}$

Both chemokines and adhesion molecules can promote $\mathrm{T}$ cell trafficking to extraintestinal sites. The activation and expansion of effector memory $\mathrm{T}$ cells can lead to the induction of MAdCAM- 1 and CCL25 expression in the liver, thereby promoting the recruitment of $\mathrm{CCR}^{+} \alpha 4 \beta 7^{+}$mucosal T cells and development of inflammation. ${ }^{22}$ Upregulation of inflammation-related adhesion molecules and chemokines also enables the capture of effector $\mathrm{T}$ cells, facilitating recruitment to sites other than extraintestinal sites. IL-6 produced during inflammation promotes the influx of naïve and memory $\mathrm{T}$ cells by increasing the expression of intercellular adhesion molecule-1 (ICAM-1) in high endothelial venules of the reactive lymph nodes. ${ }^{23}$ ICAM-1 can also be upregulated with pre-inflammatory stimuli in response to TNF- $\alpha$, IL- $1 \beta$, and interferon- $\gamma$. $^{24}$

Neutrophils altered by inflammatory cytokines can enhance their response upon subsequent activation, referred to as "neutrophil priming." ${ }^{25}$ Neutrophils in patients with gut inflammation are primed to secrete high levels of TNF- $\alpha$ and IL$1 \beta$ after stimulation with lipid polysaccharides. ${ }^{26}$ However, patients with CD have a defective neutrophil recruitment after the introduction of subcutaneous bacteria, which severely delays the subsequent clearance of these bacteria. ${ }^{27}$ In addition to changes in circulating monocytes and macrophages in patients with IBD, reduced inflammatory cytokine production in response to bacterial stimuli has also been reported. ${ }^{27,28} \mathrm{Neu}-$ trophil-platelet interactions play an important role in the degree of tissue neutrophil infiltration through neutrophil integrins $\left(\alpha_{1} \beta_{2}\right.$ and $\left.\alpha_{\mathrm{M}} \beta_{2}\right)$ and P-selectin glycoprotein ligand 1; however, neutrophil priming and/or activation in the peripheral site are inhibited by sphingosine. ${ }^{29,30}$ Ceramide synthase 2 (CerS2) and CerS6 mutually control the aspects of neutrophil recruitment to tissues, and chemotaxis is also regulated by sphingosine-1-phosphate through IL-8 production. In the gut of patients with SpA, IL-23 polarization does not appear to be accompanied by Th17 polarization. However, IL-23 can induce gut inflammation and may be associated with the expansion and activation of innate immune cells such as ILC3, $\gamma \delta \mathrm{T}$ cells, and mucosal-associated invariant T cells. ${ }^{11,31,32}$ Augmented expression of IL-23R in $\gamma / \delta$ T cells was also associated with enhanced IL-17 secretion. Stimulation of $\gamma / \delta$ T cells from SpA patients with IL-23 and/or anti-CD3/CD28 strongly induced polarization for IL-17 production.

\section{Autoantibodies}

On the endothelial side, pre-incubation of the synovial sections with monoclonal antibodies against ICAM- 1 and vascular adhesion protein-1 (VAP-1) showed significant inhibition of the binding of small lymphocytes from the IBD gut to the joint vessels. ${ }^{33}$ Small lamina propria lymphocytes isolated from the intestine of IBD patients adhered to the synovial vessels using VAP-1 and their endothelial ligands (CD18 \& ICAM1 , and $\alpha 4 \beta 7 / \alpha 4 \beta 1$ integrins and vascular cell adhesion molecule 1 [VCAM-1]). VAP-1 also plays a pivotal role in the recruitment and transmigration of lymphocytes via the hepatic sinusoidal endothelium, the expression of which is upregulated by inflammation. ${ }^{34}$ VCAM-1 selectively binds lymphocytes thr- 
ough integrin $\alpha 4 \beta 1$. It also mediates lymphocyte homing to extraintestinal sites such as the lung, skin, and central nervous systems with chronic inflammation. It is not usually expressed by the intestinal venules but can be upregulated in the blood vessels during inflammation. ${ }^{35}$ CCR10 with integrin $\alpha 4 \beta 1$ and VCAM- 1 also mediate the localization of integrin $\alpha 4 \beta 7$-negative plasmablasts to non-intestinal mucosal sites (salivary and lacrimal glands and respiratory and urogenital tracts) where CCL28 is also expressed. Among the effector and central memory T cells, CCR10 is limited to integrin $\alpha 4 \beta 1^{+} \alpha 4 \beta 1^{-}$cutaneous lymphocyte antigen ${ }^{+}$skin homing cells (which provide $\mathrm{T}$ cell attraction to the CCR10 ligand CCL27 expressed in keratinocytes). Inflammation or mechanical stress in the extraintestinal sites can lead to the recruitment of gut-producing effector $\mathrm{T}$ cells and further increase the inflammatory process. $^{34,36}$

In addition, molecular mimicry, peptide sequences between the gut bacteria and host major histocompatibility complex (MHC) molecules, and reactivation by cross-reactive components of microbiomes or host antigens have been reported. $^{37,38}$

Peptides expressing common epitopes shared by colons and extra-colonic organs, autoimmune responses to the isoform of tropomyosin (tropomyosin related peptide) expressed in the eyes, skin, joints, biliary epithelia, and intestines can help clarify the pathogenesis of EIMs. ${ }^{39,40}$ Bacteria can translocate across the leaky epithelial barriers and cause adaptive immune responses that are indistinguishable from bacterial epitopes in joints or skin. There is still a lack of clear evidence on the role of circulating antibodies or immune complexes in the pathogenesis of EIMs.

In a mice model, retina-specific $\mathrm{T}$ cells causing human blindness due to uveitis received microbial-dependent activation signals, most likely cross-reactive bacterial antigens. ${ }^{41}$ This result showed that the activation of autoreactive $\mathrm{T}$ cells via gut microbes may drive uveitis. In another mice model of autoimmune uveitis, enhanced leukocytes trafficking between the intestine and eyes were observed. ${ }^{42}$ The antigen specificity of $\mathrm{T}$ cells responsible for EIMs in humans will require further confirmation.

\section{Genetics of EIMs}

Genetic risk loci for both IBD and EIMs overlap extensively. PSC genes and their linked IBD genes are involved in T cell apoptosis (UBASH3A, BCL2L11, FOXO1, and IRF8) and the Janus kinase/signal transducers and activators of transcription
(JAK/STAT) pathways signaling pathways (SOCS1, JAK2, STAT3, and TYK2). In case of AS, T cell apoptosis pathways interact with the IBD genes. The AS-related genes, TAPBPL and NPEPPS, function similarly to the AS covalent gene ERAP1 in patients with IBD, which is important for both the development of AS and IBD. ERAP1 variant K528R associated with $\mathrm{CD}$ has involved in aberrant peptide production resulting in suboptimal peptide MHC I complex. ${ }^{43}$ The CD risk gene, NOD2/CARD15, which encodes a pattern recognition receptor, is also associated with sacroiliitis and uveitis. ${ }^{44,45}$ STAT4 is also responsible for the expansion of Th17 cells activated by IL-23, which promotes chronic inflammation in innate and adaptive immunity and is considerably associated with the development of EIMs involving the joints and eyes in patients with IBD ${ }^{46}$ However, it is still unknown whether all relevant loci contribute specifically to the pathology of EIMs or whether there are genes that generally cause EIMs by not restricting the inflammatory response to specific body compartments. Therefore, further research is needed.

\section{CLINICAL ASPECTS OF EIMs}

This following section describes the paradigms and tools currently available in clinical studies of the musculoskeletal/joint, skin, eye, vessels, and hepatobiliary EIMs (Table 2).

\section{Musculoskeletal/Joint Manifestations}

Inflammatory arthrosis of IBD is the most common EIM, with a prevalence of $20 \%-50 \%$ for axial inflammation and $5 \%-20 \%$ for peripheral arthritis. ${ }^{47}$ Peripheral arthritis in patients with IBD is mainly asymmetric, oligo-articular, and is predominantly found in CD. IBD-related joint symptoms can be subdivided into non-inflammatory and inflammatory joint pain. Arthralgia is a major medical problem in patients with IBD. Compared to patients with IBD without arthralgia, non-inflammatory joint pain was reported in $16 \%$ of patients with a significant worsening of their HRQOL determined using the 36-Item Short-Form Health Survey and the Inflammatory Bowel Disease Questionnaire. ${ }^{48}$ The current gold standard tool for layering SpA is the Ankylosing Spondylitis Disease Activity Score, which can measure the disease activity that may be followed over time..$^{49}$ In general, the prognosis for peripheral arthritis is good. The prognosis for axial involvement is less favorable and is not related to the prognosis of IBD but to the progression of IBD. 
Table 2. Diagnosis of Extraintestinal Manifestations

\begin{tabular}{|c|c|c|}
\hline System & Extraintestinal manifestations & Diagnosis \\
\hline \multirow[t]{2}{*}{$\begin{array}{l}\text { Musculoskeletal/ } \\
\text { joint }\end{array}$} & Spondyloarthritis & $\begin{array}{l}\text { Assessment of SpondyloArthritis International Society modified Berlin algorithm } \\
\text { Without radiographic sacroiliitis } \\
\text { HLA-B27 testing } \\
\text { Evaluated for sacroiliitis by MRI }\end{array}$ \\
\hline & Ankylosing spondylitis & $\begin{array}{l}\text { Modified New York Classification criteria ( } \geq 1 \text { radiological and } \geq 1 \text { clinical) } \\
\text { Clinical criteria } \\
\text { Back pain and stiffness ( } \geq 3 \text { months), improves with exercise, but is not relieved by rest } \\
\text { Limitation of motion of the lumbar spine in both the sagittal and frontal planes } \\
\text { Limitation of chest expansion relative to normal values correlated for age and sex } \\
\text { Radiological criterion } \\
\text { Image of sacroiliitis (grade } \geq 2 \text { bilaterally or grade } \geq 3 \text { unilaterally) }\end{array}$ \\
\hline \multirow[t]{4}{*}{ Skin } & Erythema nodosum & $\begin{array}{l}\text { Patient history and physical examination } \\
\text { Skin biopsy }\end{array}$ \\
\hline & Pyoderma gangrenosum & $\begin{array}{l}\text { Diagnostic criteria ( } \geq 1 \text { major \& } \geq 4 \text { minor) } \\
\text { Biopsy of ulcer edge demonstrating neutrophilic infiltrate } \\
\text { Biopsy of ulcer edge } \\
\text { Minor criteria } \\
\text { Exclusion of infection } \\
\text { Pathergy } \\
\text { Personal history and history of papule, pustule, or vesicle } \\
\text { Peripheral erythema, undermining border, and tenderness at site of ulceration } \\
\text { Multiple ulcerations ( } \geq 1 \text { anterior lower leg) } \\
\text { Cribriform or "wrinkled paper" scar(s) } \\
\text { Decrease in ulcer size within } 1 \text { month of initiating immunosuppressive medications }\end{array}$ \\
\hline & Psoriasis & $\begin{array}{l}\text { History and physical examination (Koebner phenomenon) } \\
\text { Skin biopsy (punch biopsy) }\end{array}$ \\
\hline & Eczema & $\begin{array}{l}\text { Diagnostic criteria } \\
\text { Evidence of pruritic skin, including the report by a parent of a child rubbing or scratching } \\
\text { In addition to itchy skin, } 3 \text { or more of the following are needed to make the diagnosis: } \\
\text { History of skin, asthma or hay fever } \\
\text { Dry skin within the past year } \\
\text { Symptoms beginning in a child ( }<2 \text { years of age) } \\
\text { Visible dermatitis involving flexural surfaces }\end{array}$ \\
\hline \multirow[t]{2}{*}{ Eye } & Episcleritis/scleritis & $\begin{array}{l}\text { History and physical examination } \\
\text { Slit-lamp biomicroscopy (ophthalmologist) }\end{array}$ \\
\hline & Uveitis (anterior or posterior) & $\begin{array}{l}\text { History and physical examination } \\
\text { Slit-lamp biomicroscopy (ophthalmologist) }\end{array}$ \\
\hline \multirow[t]{3}{*}{ Cardiovascular } & Infective endocarditis & $\begin{array}{l}\text { Fever } \pm \text { relevant cardiac risk factors or other predisposing conditions } \\
\text { Modified Duke criteria } \\
E C G, C T, T E, T E E \text {, or chest X-ray }\end{array}$ \\
\hline & Takayasu's arteritis & $\begin{array}{l}\text { Clinical findings } \\
\text { MRA or CT angiography }\end{array}$ \\
\hline & Pericarditis & ECG, echocardiography or chest X-ray \\
\hline \multirow{2}{*}{$\begin{array}{l}\text { Hepato-pancreato- } \\
\text { biliary }\end{array}$} & PSC & MRCP, ERCP, PTC \\
\hline & NAFLD & $\begin{array}{l}\text { History and rule out other disorders } \\
\text { US, vibration controlled transient elastography } \\
\text { CT, MRI or MRS } \\
\text { Liver biopsy }\end{array}$ \\
\hline Coagulopathy & Thromboembolism & $\begin{array}{l}\text { Compression ultrasonography with Doppler } \\
\text { CT }\end{array}$ \\
\hline
\end{tabular}

HLA-B27, human leukocyte antigen-B27; ECG, electrocardiography; TTE, transthoracic echocardiography; TEE, transesophageal echocardiography; MRA, magnetic resonance angiography; $P S C$, primary sclerosing cholangitis; $M R C P$, magnetic resonance cholangio pancreatography; $E R C P$, endoscopic retrograde cholangiopancreatography; PTC, percutaneous transhepatic cholangiography; NAFLD, nonalcoholic fatty liver disease; US, ultrasound; MRS, magnetic resonance spectroscopy. 


\section{Skin Manifestations}

Diagnoses of skin manifestations are primarily based on clinical examinations, and skin biopsies can help in atypical cases. EN is associated with IBD activity, arthralgia, or fatigue. The prevalence of EN appears higher in patients with CD than in those with UC. ${ }^{50}$ EN usually appears on the extensor surface of the lower extremities, mostly in the anterior tibial region, ${ }^{51}$ and is associated with IBD flares but not always with severity, is self-limited, and usually resolves itself without the formation of ulcers or scarring. ${ }^{52}$ The appearance of nodules parallels intestinal activity, which rarely occurs before the onset of IBD or in the quiescent phase of IBD. IBD treatment leads to the resolution of cutaneous lesions. PG can occur anywhere in the body but occurs most commonly in the shin and adjacent to stomas. PG usually has a skin trauma known as "pathergy." This trauma might even be minimal, such as a venipuncture. Controversy exists over whether PG coexists with disease activity. In this case, treatment can be concentrated on the underlying intestinal disease. Patients often show a rapid response, with a stiffening of the lesions within 24 hours of treatment.

Due to the absence of specific tests for diagnosing PG, physicians can determine whether clinical and histopathological images are consistent with PG, excluding other pustules or ulcerative dermatitis. A further development of assessment tools and standardized classifications are needed.

\section{Eye Manifestations}

The most common ocular EIMs are dry eye, blepharitis, episcleritis, or anterior uveitis. Some ocular manifestations in IBD can be secondary to treatment and/or effects of the IBD itself. Ophthalmic complications usually originate from inflammation, but some of these complications may reflect the overall disease activity. ${ }^{53}$ Episcleritis can be unilateral or bilateral and is more common in women. It usually does not need specific treatments other than those for the original disease. Scleritis is chronic and is characterized by edema and cellular infiltration of the scleral tissues. Uveitis is not common but can have potentially more serious consequences. It occurs acutely or subacutely and is usually very painful. Anterior uveitis is also commonly referred to as iritis, which indicates pain and photophobia and can be associated with blurred vision or floaters. The slit-lamp test confirms the diagnosis of an ocular EIM. In case of vision impairment, the patient must be immediately referred to the ophthalmologist to avoid vision loss.

\section{Vascular Manifestations}

Cardiovascular manifestations in IBD mostly occur as an immune-related pathogenesis including pericarditis, myocarditis, venous and arterial thromboembolisms, arrhythmias and conduction disorders, infective endocarditis, and Takayasu's arteritis. Pericarditis is the most frequent cardiovascular EIM in patients with IBD but has not been associated with IBD activity. ${ }^{54}$ The clinical picture of myocarditis or pericarditis related to IBD is nonspecific. These patients may have symptoms of an acute coronary syndrome, heart failure, or arrhythmias and may also have cardiogenic shock or sudden death.

If this clinical picture occurs within the first 28 days after starting treatment with 5-aminosalicylic acid or its derivatives, drug toxicity is suspected. ${ }^{55}$ Because 12-lead electrocardiograms and biomarkers of cardiac injury are also nonspecific, transthoracic echocardiography, coronary angiography, or cardiovascular magnetic resonance imaging may offer more precise information for all patients with clinical presentations suggesting myopericarditis. ${ }^{56}$ Furthermore, endomyocardial biopsies can be considered a gold standard for the diagnosis of myocarditis. ${ }^{57}$

In meta-analysis studies, the risk of arterial thromboembolic events or cardiovascular mortality in patients with IBD does not increase but is associated with an increase in the risk of cardiovascular morbidities from cerebrovascular accidents and ischemic heart disease, especially in women. ${ }^{58,59}$ Lower rates of peripheral vascular disease in IBD do not appear to increase the risk of peripheral arterial thromboembolic diseases. However, hyperhomocysteinemia, a risk factor for arterial and venous thrombosis, is more common in patients with IBD than in the general population. ${ }^{60}$ In addition, an increased risk of arterial thrombosis due to systemic inflammation and hypercoagulability involves premature atherosclerosis. ${ }^{61}$

\section{Hepatobiliary Manifestations}

Approximately $20 \%$ to $30 \%$ of patients with IBD show abnormal hepatic biochemistries. ${ }^{62}$ PSC is highly correlated with IBD, and about $50 \%-80 \%$ of patients with PSC have an accompanying IBD. ${ }^{63}$ Patients with IBD and PSC often have predominantly right-sided, high-frequency "backwash" ileitis, and rectal sparing. ${ }^{64}$ Patients with UC, when diagnosed with PSC, are negatively associated with smoking and older ages. ${ }^{65}$ Symptoms are intermittent and can be confusing due to the underlying IBD symptoms. In patients with IBD, PSC is usually asymptomatic, which requires an awareness of high clinical association. Although typically imaged using magnetic reso- 
Table 3. Treatments for Extraintestinal Manifestations

\begin{tabular}{|c|c|c|c|c|c|c|c|}
\hline System & $\begin{array}{l}\text { Extraintestinal } \\
\text { manifestations }\end{array}$ & NSAIDs & Corticosteroids & 5-ASA & MTX/AZA & $\begin{array}{l}\text { Immuno- } \\
\text { modulators }\end{array}$ & Anti-TNFs \\
\hline \multirow[t]{3}{*}{ Musculoskeletal/joint } & Spondyloarthritis & $\bigcirc$ & - & $\bigcirc$ & - & - & $\bigcirc$ \\
\hline & Ankylosing spondylitis & $\bigcirc$ & - & $\triangle$ & $\triangle$ & - & $\bigcirc$ \\
\hline & Peripheral arthritis & $\bigcirc$ & $\bigcirc$ & (C) & $\bigcirc$ & - & $\bigcirc$ \\
\hline \multirow[t]{2}{*}{ Skin } & Erythema nodosum & O & (O) & - & - & - & $\bigcirc$ \\
\hline & Pyoderma gangrenosum & - & (O) & - & - & $\bigcirc$ & $\bigcirc$ \\
\hline \multirow[t]{2}{*}{ Eye } & Episcleritis/scleritis & $\bigcirc$ & $\bigcirc$ & - & $\triangle$ & - & $\triangle$ \\
\hline & Uveitis & - & $\bigcirc$ & - & $\triangle$ & $\triangle$ & $\triangle$ \\
\hline \multirow[t]{3}{*}{ Hepatobiliary } & PSC & \multicolumn{6}{|c|}{ Ursodeoxycholic acid, ERCP and liver transplantation } \\
\hline & Autoimmune hepatitis & \multicolumn{6}{|c|}{ Prednisolone alone or in combination with azathioprine } \\
\hline & Granulomatous hepatitis & - & $\bigcirc$ & - & - & $\bigcirc$ & $\bigcirc$ \\
\hline \multirow[t]{3}{*}{ Coagulopathy } & Deep vein thrombosis & \multicolumn{6}{|c|}{ Low-molecular-weight heparin, low-dose unfractionated heparin or fondaparinux } \\
\hline & Pulmonary embolism & \multicolumn{6}{|c|}{ Vitamin K antagonist (acenocoumarol, warfarin, fluindione) } \\
\hline & Thromboembolism & \multicolumn{6}{|c|}{ Non-vitamin K antagonist oral anticoagulants (dabigatran, rivaroxaban, apixaban, and edoxaban) } \\
\hline
\end{tabular}

5-ASA, 5-aminosalicylic acid; MTX, methotrexate; AZA, azathioprine; PSC, primary sclerosing cholangitis; ERCP, endoscopic retrograde cholangiopancreatography; $\bigcirc$, recommended; $\bigcirc$, available; $\triangle$, controversial.

nance cholangiography and confirmed using endoscopic retrograde cholangiopancreatography (ERCP), the Crohn's and Colitis Organisation consensus group suggests that ERCP be performed only for intervention in stricture dilatation and/or if brush cytology specimen sampling is indicated. ${ }^{66}$

Autoimmune hepatitis (AIH)/PSC overlapping syndrome is seen in UC patients than $\mathrm{CD} .{ }^{67}$ It is suspected that the characteristics of AIH and PSC in the same patient are present, and a definitive diagnosis of AIH is required according to the international AIH group criteria. ${ }^{68}$

The prevalence of AIH in pediatric IBD patients is $1.6 \%{ }^{69}$ AIH can be divided into 2 types. AIH type 1 is positive for antinuclear antibodies and/or smooth muscle antibodies, whereas AIH type 2 is liver/kidney microsomal type 1 and/or antiliver cytosol type 1 is positive. Even if antinuclear antibodies or smooth muscle antibodies is 1:20 or liver/kidney microsomal type 1 is 1:10 in children, it is associated with AIH in children. $^{70}$

Granulomatous hepatitis is a rare EIM of CD. The estimated prevalence is less than $1 \%$ of IBD. It may manifest fever, alkaline phosphatase, hepatosplenomegaly. Granulomatous hepatitis may be induced by mesalazine and sulfasalazine therapy. ${ }^{71}$

In nonalcoholic fatty liver disease, IBD-specific risk factors include intraperitoneal abscesses, fistula disease, colitis severity, malnutrition, protein loss, and drug use.

Thiopurine and gallstones are the most common causes of acute pancreatitis in patients with IBD, and acute pancreatitis caused by thiopurine is generally not complicated and is selflimiting. ${ }^{72}$ Most chronic pancreatitis cases in patients with IBD are idiopathic, and PSC is a less common cause. Exocrine pancreatic insufficiency appears to be the most common pancreatic symptom in IBD, but its clinical significance remains unknown.

\section{TREATMENT FOR EIMS INCLUDING BIOLOGICS}

Optimizing treatments is often challenging. There is lack of high-quality evidence to support the most commonly used treatment methods for EIMs and other treatment strategies that can complement them. Treatment of EIMs should be based on the severity of symptoms and their association with IBD activity. The primary goal is symptom control and preservation of mobility and function. In some cases, symptomatic treatment with physiotherapy may be sufficient (Table 3).

\section{Joint Manifestations}

Treatment of IBD-related arthropathy is based on SpA studies, predominantly AS. Patients with axial SpA should be co-managed by rheumatologists because of the potentially disruptive disease progress. Severity of type 1 arthritis usually runs parallel to IBD activity, and type 2 arthritis usually requires longterm treatment. Therefore, effective treatment of underlying 
gut inflammation is important for the treatment of peripheral arthritis.

Intensive physiotherapy may be helpful in AS, and NSAIDs should be used for a short time period due to their potential to activate underlying IBD. ${ }^{73}$ Cyclooxygenase-2 inhibitors such as etoricoxib and celecoxib may be safer and have a lower risk of disease flare than conventional NSAIDs. ${ }^{74}$ Sulfasalazine, methotrexate (MTX), and azathioprine are generally ineffective or only slightly effective in the treatment of AS. However, sulfasalazine is effective in treating patients with peripheral joint disease, especially those with a short disease duration. ${ }^{75}$ Sulfasalazine is an optional treatment in patients with $\mathrm{SpA}$ and peripheral disease and may be effective for large joint arthropathy. ${ }^{76}$ MTX and azathioprine are considered marginally effective in the treatment of peripheral arthropathy. For patients with intolerance, unresponsiveness, or poor response to NSAIDs, anti-TNF therapy is the preferred treatment because of the limited efficacy of MTX and thiopurine. ${ }^{77}$

\section{Skin Manifestations}

Treatment of EN consists of treating the underlying IBD, and systemic steroids may be helpful. For patients with resistant or frequent relapses, immunomodulators and anti-TNF therapy can be considered alone or in combination. ${ }^{78}$

PG treatment strategies are the same between IBD and nonIBD patients. Systemic corticosteroids are considered as the first-line treatment, and oral or intravenous tacrolimus with oral cyclosporines may be considered for refractory cases. ${ }^{79,80}$ Anti-TNF therapy represents a new and effective treatment option for medically refractory PG cases.

\section{Eye Manifestations}

Due to the potential loss of vision, ophthalmologists must evaluate each suspected case to confirm the diagnosis and help guide the treatment. Dry eyes may be treated with topical lubricants. Episcleritis may be self-limiting and usually responds to underlying IBD treatment along with topical NSAIDs and glucocorticoids. ${ }^{81}$ Uveitis requires urgent consultations with ophthalmologists and needs prompt treatment because of the risk of vision loss. Treatment usually consists of steroids. Other therapies (azathioprine, MTX, or anti-TNF therapy) have been discussed only for resistant cases. ${ }^{82}$

\section{Vascular Manifestations}

Because thromboembolism occurs due to multifactorial causes and acquired risk factors, precautions (hydration, cor- rection of vitamin deficiencies, and wearing gradual compression stockings) can help prevent venous thromboembolisms (VTEs). Prophylactic anticoagulation therapy is recommended for high-risk patients (hospitalized due to an active phase or surgery) with VTEs. ${ }^{83}$ There is still no evidence for the prophylaxis of active disease in outpatients with IBD. However, because many VTEs occur in outpatients with IBD, guidelines are required for VTE prophylaxis when patients with IBD have acutely or chronically active diseases. Acute deep vein thromboses and pulmonary embolisms are treated using anticoagulants according to international guidelines. Low-molecularweight heparin is commonly used, and low-dose unfractionated heparin or fondaparinux can be used as an alternative option. Long-term treatment includes oral anticoagulants such as vitamin $\mathrm{K}$ antagonists (acenocoumarol, warfarin, fluindione) or non-vitamin $\mathrm{K}$ antagonist oral anticoagulants, such as dabigatran, rivaroxaban, apixaban, or edoxaban.

\section{Hepatobiliary Manifestations}

Ursodeoxycholic acid improves abnormal liver function tests and is widely used in patients with PSC, but prognosis improvements are controversial. Other therapeutic agents (steroid, cyclosporine, tacrolimus, MTX, or anti-TNF therapy) still lack evidence for improvements in clinical endpoints. ${ }^{84}$ ERCPs play a role in the management of biliary strictures and should be performed after antibiotic prophylaxis in patients with PSC. It can reduce the risk of cholangitis, bacteremia, and septicemia after ERCP. ${ }^{85}$ Patients with PSC and end-stage liver disease should be evaluated to determine whether liver transplantations should be performed according to the standard guidelines. PSC has a significantly greater risk for the development of colorectal dysplasia and carcinoma in patients with UC. ${ }^{86}$ Therefore, surveillance colonoscopies with target biopsies are recommended at the diagnosis and at every 1-2 years thereafter. $^{87}$

Immunosuppressive therapy with prednisolone alone or in combination with azathioprine is the main therapeutic regimen of AIH and has been to show the prolongation of life expectancy. ${ }^{67}$ In granulomatous hepatitis, corticosteroids and immunosuppressive drugs are used for treatment.

\section{Biologics and Probiotics for EIMs}

Biologics have been tested to prove their efficacy in the treatment of EIMs as well as IBD. Clinical benefits of infliximab or adalimumab may not be limited to gut treatment effects but may extend to improve EIMs ${ }^{88}$ Infliximab and adalimumab 
therapy also have potential beneficial effects on metabolic bone diseases, hematologic diseases, or vascular EIMs. ${ }^{88}$ However, anti-TNF treatments can cause paradoxical cutaneous inflammations, which are considered a class drug effect, and are generally reversible upon drug transition or discontinuation. This paradoxical cutaneous inflammation manifests itself or appears in most cases as psoriasis, which accounts for why anti-TNF- $\alpha$ drug use is increasingly recognized as a cause of drug-induced skin lesions. ${ }^{89}$ Paradoxical psoriasis skin lesions are characterized by selective overexpression of type I interferon, skin accumulation of plasmacytoid dendritic cells (pDCs), and reduced T cell counts. ${ }^{90}$ Anti-TNF- $\alpha$ treatment prolongs the production of type 1 interferons by pDC by inhibiting pDC maturation. Type I interferon overexpression results in a skin phenotype of paradoxical psoriasis, independent of T cells, unlike classical psoriasis. TNF- $\alpha$ inhibition may reduce the accumulation of Thl and Th17 cells at the site of inflammation but may induce compensatory expansion at other locations, resulting in skin lesions. ${ }^{91}$

The role of vedolizumab in EIM treatment has not been defined yet. Vedolizumab inhibits the binding of $\alpha 4 \beta 7+$ integrins to the intestinal mucosal cell adhesion molecule (MAdCAM-1) but not to VCAM- $1 .{ }^{92}$ The binding mechanism seems to be selective for cutaneous intestinal lymphocyte migration. In contrast, lymphocyte migration by VCAM-1 is unaffected. Nevertheless, the overlapping binding behavior of $\alpha_{4} \beta_{7}$ with MAdCAM- 1 and VCAM- 1 may be due to partly different affinities. ${ }^{9293}$ According to these results, vedolizumab has no direct therapeutic effect on cutaneous EIMs and can play a role in EIM management by treating disease activity in EIMs that parallels IBD ${ }^{94}$ In a systematic review, vedolizumab was suggested to reduce the incidence of new EIMs but also be effective in the treatment of preexisting EIMs (particularly PSC, rheumatic and cutaneous manifestations). ${ }^{95}$

Data on other biologicals are limited. Ustekinumab is a fully human IgG1 kappa monoclonal antibody that blocks the IL12/23 p40 subunit. IL-12 and IL-23 are associated with adaptive immune responses, and blocking IL-12/IL-23 helps improve chronic inflammatory conditions. Ustekinumab has been reported for the management of cutaneous and joint EIMs (psoriasis, PsA) ${ }^{96,97}$ In addition, it is effective for the treatment of anti-TNF antibody-related paradoxical cutaneous inflammation ${ }^{98}$ However, paradoxical arthralgic or cutaneous manifestations have also been reported. ${ }^{99,100}$ In addition, it did not reduce the occurrence of new EIMs. ${ }^{101}$ Therefore, ustekinumab requires additional safety and efficacy evidence from randomized controlled trials and open-label, real-world cohorts. Ustekinumab will be an important treatment option in EIMs and IBD because it is superior to vedolizumab in terms of overall efficacy, rapid onset, and efficacy in small intestine disease.

Tofacitinib is a selective JAK inhibitor and the first oral medication approved for use in treating moderate-to-severe UC. Its efficacy of treating cutaneous EIMs has been reported. STAT3 was significantly upregulated in PG/EN compared to psoriasis and revealed potential benefits from JAK inhibition in these patients. ${ }^{94,102}$ In a double-blind, head-to-head, non-inferiority, randomized controlled trial in patients with rheumatoid arthritis who had insufficient response to MTX treatment, combination therapy with tofacitinib and MTX showed similar efficacy to that with adalimumab and MTX, and tofacitinib monotherapy was shown to be inferior to either combination. ${ }^{103}$ Because tofacitinib is expected to be free of anti-drug antibodies due to its low molecular weight, it results in a rapid therapeutic effect in patients with acute severe UC. Further studies on the efficacy and safety of the combination therapy with tofacitinib and other drugs are needed. In addition, there is a need to compare the efficacy and side-effects of upadacitinib and tofacitinib, which are selective JAK1 inhibitors.

Data on probiotics for EIMs are limited. Several studies suggested that the use of probiotics has a beneficial effect for $\mathrm{cu}^{-}$ taneous or arthralgic EIMs but requires further confirmation from randomized controlled trials or systematic reviews evaluating the effects of probiotics on EIMs. ${ }^{104,105}$

\section{KEY RECOMMENDATIONS FOR IMPROVING THE DISEASE COURSE AND PRINCIPLES TO BE APPLIED TO TREATMENT}

Physicians should always consider EIMs in the management of patients with IBD. Early diagnosis and treatment can prevent permanent tissue destruction and the development of complications in patients with IBD with developing EIMs. Diagnostic delay is considered a risk factor for the development of EIM complications and abdominal surgery ${ }^{106}$ Uveitis can result in visual impairment if the diagnosis is delayed. Due to the diagnostic delay accompanying these inflammatory conditions, the need for proper approaches and tools that can quickly provide accurate and viable information is further emphasized. Sex, age, ethnicity, geography, socioeconomic status, duration, and extension of disease, disease activity, and a history of medications are associated with the diagnoses of 
EIMs. Genetic factors and dysbiosis of gut microbiomes also play an important role in identifying EIMs. Physicians should be careful because specific features of the clinical presentations may signal the possibility of future EIMs. Screening and surveillance of EIMs are difficult for physicians in only one specialty to include in their treatment protocols. Therefore, it is necessary to follow-up with multidisciplinary care. Educating patients on EIMs and their complications will improve the effectiveness of medications and follow-ups. However, research has shown that certain EIMs, such as VTEs, PSCs, and nephrolithiasis, may be less emphasized in patient education. ${ }^{107}$ Patients' perception of thromboembolisms or cardiovascular EIMs can have a significant impact on the associated morbidity and mortality. VTE has a higher incidence in patients with IBD than in the general population, and IBD disease activity is associated with an increased risk of VTE. ${ }^{108}$ However, only $12 \%$ of patients were aware that the risk of VTE in the outpatient environment was high and less than half of the patients were convinced that they recognized the signs and symptoms of deep vein thromboses and pulmonary embolisms, ${ }^{107}$ indicating a need to reconsider the awareness of EIMs in patients with IBD.

The role of mobile applicable for the self-management of IBD by patients will continue to increase by providing a more efficient, effective, and good quality healthcare system at lower costs. ${ }^{109}$ Daily monitoring of UC through TrueColours UC improved UC management while also reducing the burden placed on individual and healthcare services. ${ }^{110}$ These applications can not only remotely monitor patients via electronic patient-reported outcome measures (PROs) or device-generated data (such as activity trackers) but also improves patient engagement. ${ }^{111}$ The goal of recent IBD therapies is moving toward a therapeutic-targeted strategy using PROs, biomarkers of inflammation, and mucosal healing to optimize therapeutics and should also be used for the treatment of EIMs.

\section{CONCLUSIONS}

For EIMs of IBD, new mechanisms are being uncovered in a number of fields, including immunology, microbiology, and genetics, and can reveal a wide range of therapeutic targets. It is fundamentally important to ensure proper diagnosis and management of EIMs, which can often result in a much more debilitating and lower quality of life than actual IBD. Better patient images require new tools to monitor inflammation from various angles, and managements for complex and diverse
EIMs should be discussed, treated, and tracked through multidisciplinary care. In addition to establishing the required diagnosis and treatment process, patients should be encouraged to participate more actively in the various stages of medical care, measurements, implementations, and evaluations of the quality of their care.

\section{FINANCIAL SUPPORT}

The authors received no financial support for the research, authorship, and/or publication of this article.

\section{CONFLICT OF INTEREST}

Cheon JH has been the Editor of Intestinal Research since 2013. However, he was not involved in the peer reviewer selection, evaluation, or decision of this article. Except for that, no potential conflict of interest relevant to this article was reported.

\section{AUTHOR CONTRIBUTION}

Conceptualization, methodology, writing - original draft preparation: Kim JM. Writing - review and editing: Cheon JH. Supervision project administration: Cheon JH. Approval of final manuscript: all author.

\section{ORCID}

Kim JM https://orcid.org/0000-0003-3910-9380

Cheon JH ～https://orcid.org/0000-0002-2282-8904

\section{REFERENCES}

1. Hedin CRH, Vavricka SR, Stagg AJ, et al. The pathogenesis of extraintestinal manifestations: implications for IBD research, diagnosis, and therapy. J Crohns Colitis 2019;13:541-554.

2. Vavricka SR, Schoepfer A, Scharl M, Lakatos PL, Navarini A, Rogler G. Extraintestinal manifestations of inflammatory bowel disease. Inflamm Bowel Dis 2015;21:1982-1992.

3. Yang BR, Choi NK, Kim MS, et al. Prevalence of extraintestinal manifestations in Korean inflammatory bowel disease patients. PLoS One 2018;13:e0200363.

4. Severs M, Spekhorst LM, Mangen MJ, et al. Sex-related differences in patients with inflammatory bowel disease: results of 2 prospective cohort studies. Inflamm Bowel Dis 2018;24: 
1298-1306

5. Vavricka SR, Rogler G, Gantenbein C, et al. Chronological order of appearance of extraintestinal manifestations relative to the time of IBD diagnosis in the swiss inflammatory bowel disease cohort. Inflamm Bowel Dis 2015;21:1794-1800.

6. Wagtmans MJ, Verspaget HW, Lamers CB, van Hogezand RA. Crohn's disease in the elderly: a comparison with young adults. J Clin Gastroenterol 1998;27:129-133.

7. Zeitz J, Ak M, Müller-Mottet S, et al. Pain in IBD patients: very frequent and frequently insufficiently taken into account. PLoS One 2016;11:e0156666.

8. Scher JU, Ubeda C, Artacho A, et al. Decreased bacterial diversity characterizes the altered gut microbiota in patients with psoriatic arthritis, resembling dysbiosis in inflammatory bowel disease. Arthritis Rheumatol 2015;67:128-139.

9. Tito RY, Cypers H, Joossens M, et al. Brief report: dialister as a microbial marker of disease activity in spondyloarthritis. Arthritis Rheumatol 2017;69:114-121.

10. Viladomiu M, Kivolowitz C, Abdulhamid A, et al. IgA-coated E. coli enriched in Crohn's disease spondyloarthritis promote TH17-dependent inflammation. Sci Transl Med 2017;9: eaaf9655.

11. Ciccia F, Guggino G, Rizzo A, et al. Type 3 innate lymphoid cells producing IL-17 and IL-22 are expanded in the gut, in the peripheral blood, synovial fluid and bone marrow of patients with ankylosing spondylitis. Ann Rheum Dis 2015;74: 1739-1747.

12. Cuthbert RJ, Fragkakis EM, Dunsmuir R, et al. Brief report: group 3 innate lymphoid cells in human enthesis. Arthritis Rheumatol 2017;69:1816-1822.

13. Kummen M, Holm K, Anmarkrud JA, et al. The gut microbial profile in patients with primary sclerosing cholangitis is distinct from patients with ulcerative colitis without biliary disease and healthy controls. Gut 2017;66:611-619.

14. Nakamoto N, Sasaki N, Aoki R, et al. Gut pathobionts underlie intestinal barrier dysfunction and liver T helper 17 cell immune response in primary sclerosing cholangitis. Nat Microbiol 2019;4:492-503.

15. Dodd D, Spitzer MH, Van Treuren W, et al. A gut bacterial pathway metabolizes aromatic amino acids into nine circulating metabolites. Nature 2017;551:648-652.

16. King SJ, McCole DF. Epithelial-microbial diplomacy: escalating border tensions drive inflammation in inflammatory bowel disease. Intest Res 2019;17:177-191.

17. Godefroy E, Alameddine J, Montassier E, et al. Expression of CCR6 and CXCR6 by gut-derived CD4+/CD8 $\alpha+$ T-regulatory cells, which are decreased in blood samples from patients with inflammatory bowel diseases. Gastroenterology 2018; 155:1205-1217.

18. Adams DH, Eksteen B. Aberrant homing of mucosal T cells and extra-intestinal manifestations of inflammatory bowel disease. Nat Rev Immunol 2006;6:244-251.

19. Berlin C, Berg EL, Briskin MJ, et al. Alpha 4 beta 7 integrin mediates lymphocyte binding to the mucosal vascular addressin MAdCAM-1. Cell 1993;74:185-195.

20. Svensson M, Marsal J, Ericsson A, et al. CCL25 mediates the localization of recently activated CD8alphabeta(+) lymphocytes to the small-intestinal mucosa. J Clin Invest 2002;110: 1113-1121.

21. Mora JR, von Andrian UH. T-cell homing specificity and plasticity: new concepts and future challenges. Trends Immunol 2006;27:235-243.

22. Chapman RW. Aetiology and natural history of primary sclerosing cholangitis: a decade of progress? Gut 1991;32:14331435.

23. Chen Q, Fisher DT, Clancy KA, et al. Fever-range thermal stress promotes lymphocyte trafficking across high endothelial venules via an interleukin 6 trans-signaling mechanism. Nat Immunol 2006;7:1299-1308.

24. Henninger DD, Panés J, Eppihimer M, et al. Cytokine-induced VCAM-1 and ICAM-1 expression in different organs of the mouse. J Immunol 1997;158:1825-1832.

25. Condliffe AM, Kitchen E, Chilvers ER. Neutrophil priming: pathophysiological consequences and underlying mechanisms. Clin Sci (Lond) 1998;94:461-471.

26. Nikolaus S, Bauditz J, Gionchetti P, Witt C, Lochs H, Schreiber S. Increased secretion of pro-inflammatory cytokines by circulating polymorphonuclear neutrophils and regulation by interleukin 10 during intestinal inflammation. Gut 1998;42: 470-476.

27. Smith AM, Rahman FZ, Hayee B, et al. Disordered macrophage cytokine secretion underlies impaired acute inflammation and bacterial clearance in Crohn's disease. J Exp Med 2009;206:1883-1897.

28. Sanders TJ, McCarthy NE, Giles EM, et al. Increased production of retinoic acid by intestinal macrophages contributes to their inflammatory phenotype in patients with Crohn's disease. Gastroenterology 2014;146:1278-1288.

29. Espaillat MP, Kew RR, Obeid LM. Sphingolipids in neutrophil function and inflammatory responses: mechanisms and implications for intestinal immunity and inflammation in ulcerative colitis. Adv Biol Regul 2017;63:140-155. 
30. Soehnlein O, Steffens S, Hidalgo A, Weber C. Neutrophils as protagonists and targets in chronic inflammation. Nat Rev Immunol 2017;17:248-261.

31. Ciccia F, Accardo-Palumbo A, Alessandro R, et al. Interleukin-22 and interleukin-22-producing NKp44+ natural killer cells in subclinical gut inflammation in ankylosing spondylitis. Arthritis Rheum 2012;64:1869-1878.

32. Kenna TJ, Davidson SI, Duan R, et al. Enrichment of circulating interleukin-17-secreting interleukin-23 receptor-positive $\gamma / \delta \mathrm{T}$ cells in patients with active ankylosing spondylitis. Arthritis Rheum 2012;64:1420-1429.

33. Salmi M, Jalkanen S. Human leukocyte subpopulations from inflamed gut bind to joint vasculature using distinct sets of adhesion molecules. J Immunol 2001;166:4650-4657.

34. Lalor PF, Edwards S, McNab G, Salmi M, Jalkanen S, Adams DH. Vascular adhesion protein-1 mediates adhesion and transmigration of lymphocytes on human hepatic endothelial cells. J Immunol 2002;169:983-992.

35. Soriano A, Salas A, Salas A, et al. VCAM-1, but not ICAM-1 or MAdCAM-1, immunoblockade ameliorates DSS-induced colitis in mice. Lab Invest 2000;80:1541-1551.

36. Jacques P, McGonagle D. The role of mechanical stress in the pathogenesis of spondyloarthritis and how to combat it. Best Pract Res Clin Rheumatol 2014;28:703-710.

37. Scofield RH, Kurien B, Gross T, Warren WL, Harley JB. HLAB27 binding of peptide from its own sequence and similar peptides from bacteria: implications for spondyloarthropathies. Lancet 1995;345:1542-1544.

38. Ramos M, Alvarez I, Sesma L, Logean A, Rognan D, López de Castro JA. Molecular mimicry of an HLA-B27-derived ligand of arthritis-linked subtypes with chlamydial proteins. J Biol Chem 2002;277:37573-37581.

39. Oshitani N, Watanabe K, Nakamura S, Higuchi K, Arakawa T. Extraintestinal complications in patients with ulcerative colitis. Nihon Rinsho 2005;63:874-878.

40. Bhagat S, Das KM. A shared and unique peptide in the human colon, eye, and joint detected by a monoclonal antibody. Gastroenterology 1994;107:103-108.

41. Horai R, Zárate-Bladés CR, Dillenburg-Pilla P, et al. Microbiota-dependent activation of an autoreactive $\mathrm{T}$ cell receptor provokes autoimmunity in an immunologically privileged site. Immunity 2015;43:343-353.

42. Nakamura YK, Janowitz C, Metea C, et al. Short chain fatty acids ameliorate immune-mediated uveitis partially by altering migration of lymphocytes from the intestine. Sci Rep 2017;7:11745.
43. Reeves E, James E. The role of polymorphic ERAP1 in autoinflammatory disease. Biosci Rep 2018;38:BSR20171503.

44. Martin TM, Smith JR, Rosenbaum JT. Anterior uveitis: current concepts of pathogenesis and interactions with the spondyloarthropathies. Curr Opin Rheumatol 2002;14:337341.

45. Peeters H, Vander Cruyssen B, Laukens D, et al. Radiological sacroiliitis, a hallmark of spondylitis, is linked with CARD15 gene polymorphisms in patients with Crohn's disease. Ann Rheum Dis 2004;63:1131-1134.

46. Moon CM, Cheon JH, Kim SW, et al. Association of signal transducer and activator of transcription 4 genetic variants with extra-intestinal manifestations in inflammatory bowel disease. Life Sci 2010;86:661-667.

47. De Vos M, De Keyser F, Mielants H, Cuvelier C, Veys E. Review article: bone and joint diseases in inflammatory bowel disease. Aliment Pharmacol Ther 1998;12:397-404.

48. Palm O, Moum B, Ongre A, Gran JT. Prevalence of ankylosing spondylitis and other spondyloarthropathies among patients with inflammatory bowel disease: a population study (the IBSEN study). J Rheumatol 2002;29:511-515.

49. van der Heijde D, Lie E, Kvien TK, et al. ASDAS, a highly discriminatory ASAS-endorsed disease activity score in patients with ankylosing spondylitis. Ann Rheum Dis 2009;68:18111818.

50. Nguyen GC, Torres EA, Regueiro M, et al. Inflammatory bowel disease characteristics among African Americans, Hispanics, and non-Hispanic Whites: characterization of a large North American cohort. Am J Gastroenterol 2006;101: 1012-1023.

51. Greenstein AJ, Janowitz HD, Sachar DB. The extra-intestinal complications of Crohn's disease and ulcerative colitis: a study of 700 patients. Medicine (Baltimore) 1976;55:401-412.

52. Trost LB, McDonnell JK. Important cutaneous manifestations of inflammatory bowel disease. Postgrad Med J 2005; 81:580-585.

53. Hopkins DJ, Horan E, Burton IL, Clamp SE, de Dombal FT, Goligher JC. Ocular disorders in a series of 332 patients with Crohn's disease. Br J Ophthalmol 1974;58:732-737.

54. Kupferschmidt H, Langenegger T, Krähenbühl S. Pericarditis in chronic inflammatory bowel disease: underlying disease or side effects of therapy? Clinical problem solving. Schweiz Med Wochenschr 1996;126:2184-2190.

55. Brown G. 5-Aminosalicylic acid-associated myocarditis and pericarditis: a narrative review. Can J Hosp Pharm 2016;69: 466-472. 
56. Kindermann I, Barth C, Mahfoud F, et al. Update on myocarditis. J Am Coll Cardiol 2012;59:779-792.

57. Cooper LT, Baughman KL, Feldman AM, et al. The role of endomyocardial biopsy in the management of cardiovascular disease: a scientific statement from the American Heart Association, the American College of Cardiology, and the European Society of Cardiology. Endorsed by the Heart Failure Society of America and the Heart Failure Association of the European Society of Cardiology. J Am Coll Cardiol 2007;50:19141931.

58. Fumery M, Xiaocang C, Dauchet L, Gower-Rousseau C, Peyrin-Biroulet L, Colombel JF. Thromboembolic events and cardiovascular mortality in inflammatory bowel diseases: a meta-analysis of observational studies. J Crohns Colitis 2014;8:469-479.

59. Singh S, Singh H, Loftus EV Jr, Pardi DS. Risk of cerebrovascular accidents and ischemic heart disease in patients with inflammatory bowel disease: a systematic review and metaanalysis. Clin Gastroenterol Hepatol 2014;12:382-393.

60. Oussalah A, Guéant JL, Peyrin-Biroulet L. Meta-analysis: hyperhomocysteinaemia in inflammatory bowel diseases. Aliment Pharmacol Ther 2011;34:1173-1184.

61. Tan VP, Chung A, Yan BP, Gibson PR. Venous and arterial disease in inflammatory bowel disease. J Gastroenterol Hepatol 2013;28:1095-1113.

62. Mendes FD, Levy C, Enders FB, Loftus EV Jr, Angulo P, Lindor KD. Abnormal hepatic biochemistries in patients with inflammatory bowel disease. Am J Gastroenterol 2007;102: 344-350.

63. Fausa O, Schrumpf E, Elgjo K. Relationship of inflammatory bowel disease and primary sclerosing cholangitis. Semin Liver Dis 1991;11:31-39.

64. Loftus EV Jr, Harewood GC, Loftus CG, et al. PSC-IBD: a unique form of inflammatory bowel disease associated with primary sclerosing cholangitis. Gut 2005;54:91-96.

65. Park YE, Cheon JH, Park JJ, et al. Risk factors and clinical courses of concomitant primary sclerosing cholangitis and ulcerative colitis: a Korean multicenter study. Int J Colorectal Dis 2018;33:1497-1500.

66. Harbord M, Annese V, Vavricka SR, et al. The first European evidence-based consensus on extra-intestinal manifestations in inflammatory bowel disease. J Crohns Colitis 2016;10:239254.

67. Woodward J, Neuberger J. Autoimmune overlap syndromes. Hepatology 2001;33:994-1002.

68. Gregorio GV, Portmann B, Karani J, et al. Autoimmune hepa- titis/sclerosing cholangitis overlap syndrome in childhood: a 16-year prospective study. Hepatology 2001;33:544-553.

69. Jose FA, Garnett EA, Vittinghoff E, et al. Development of extraintestinal manifestations in pediatric patients with inflammatory bowel disease. Inflamm Bowel Dis 2009;15:63-68.

70. Manns MP, Czaja AJ, Gorham JD, et al. Diagnosis and management of autoimmune hepatitis. Hepatology 2010;51:21932213.

71. Braun M, Fraser GM, Kunin M, Salamon F, Tur-Kaspa R. Mesalamine-induced granulomatous hepatitis. Am J Gastroenterol 1999;94:1973-1974.

72. Ramos LR, Sachar DB, DiMaio CJ, Colombel JF, Torres J. Inflammatory bowel disease and pancreatitis: a review. J Crohns Colitis 2016;10:95-104.

73. Klein A, Eliakim R. Non steroidal anti-inflammatory drugs and inflammatory bowel disease. Pharmaceuticals (Basel) 2010;3:1084-1092.

74. El Miedany Y, Youssef S, Ahmed I, El Gaafary M. The gastrointestinal safety and effect on disease activity of etoricoxib, a selective cox-2 inhibitor in inflammatory bowel diseases. Am J Gastroenterol 2006;101:311-317.

75. Chen J, Liu C. Sulfasalazine for ankylosing spondylitis. Cochrane Database Syst Rev 2005;(2):CD004800.

76. Dougados M, vam der Linden S, Leirisalo-Repo M, et al. Sulfasalazine in the treatment of spondyloarthropathy: a randomized, multicenter, double-blind, placebo-controlled study. Arthritis Rheum 1995;38:618-627.

77. Baraliakos X, Braun J. Biologic therapies for spondyloarthritis: what is new? Curr Rheumatol Rep 2012;14:422-427.

78. Kaufman I, Caspi D, Yeshurun D, Dotan I, Yaron M, Elkayam $O$. The effect of infliximab on extraintestinal manifestations of Crohn's disease. Rheumatol Int 2005;25:406-410.

79. Baumgart DC, Wiedenmann B, Dignass AU. Rescue therapy with tacrolimus is effective in patients with severe and refractory inflammatory bowel disease. Aliment Pharmacol Ther 2003;17:1273-1281.

80. Brooklyn T, Dunnill G, Probert C. Diagnosis and treatment of pyoderma gangrenosum. BMJ 2006;333:181-184.

81. Mintz R, Feller ER, Bahr RL, Shah SA. Ocular manifestations of inflammatory bowel disease. Inflamm Bowel Dis 2004;10: 135-139.

82. Fries W, Giofré MR, Catanoso M, Lo Gullo R. Treatment of acute uveitis associated with Crohn's disease and sacroileitis with infliximab. Am J Gastroenterol 2002;97:499-500.

83. Nguyen GC, Bernstein CN, Bitton A, et al. Consensus statements on the risk, prevention, and treatment of venous 
thromboembolism in inflammatory bowel disease: Canadian Association of Gastroenterology. Gastroenterology 2014; 146:835-848

84. Chandok N, Hirschfield GM. Management of primary sclerosing cholangitis: conventions and controversies. Can J Gastroenterol 2012;26:261-268.

85. Brand M, Bizos D, O’Farrell P Jr. Antibiotic prophylaxis for patients undergoing elective endoscopic retrograde cholangiopancreatography. Cochrane Database Syst Rev 2010;(10): CD007345.

86. Soetikno RM, Lin OS, Heidenreich PA, Young HS, Blackstone MO. Increased risk of colorectal neoplasia in patients with primary sclerosing cholangitis and ulcerative colitis: a metaanalysis. Gastrointest Endosc 2002;56:48-54.

87. Van Assche G, Dignass A, Bokemeyer B, et al. Second European evidence-based consensus on the diagnosis and management of ulcerative colitis part 3: special situations. J Crohns Colitis 2013;7:1-33.

88. Peyrin-Biroulet L, Van Assche G, Gómez-Ulloa D, et al. Systematic review of tumor necrosis factor antagonists in extraintestinal manifestations in inflammatory bowel disease. Clin Gastroenterol Hepatol 2017;15:25-36.

89. de Gannes GC, Ghoreishi M, Pope J, et al. Psoriasis and pustular dermatitis triggered by TNF-\{alpha\} inhibitors in patients with rheumatologic conditions. Arch Dermatol 2007; 143:223-231.

90. Conrad C, Di Domizio J, Mylonas A, et al. TNF blockade induces a dysregulated type I interferon response without autoimmunity in paradoxical psoriasis. Nat Commun 2018;9:25.

91. Greuter T, Navarini A, Vavricka SR. Skin manifestations of inflammatory bowel disease. Clin Rev Allergy Immunol 2017; 53:413-427.

92. Soler D, Chapman T, Yang LL, Wyant T, Egan R, Fedyk ER. The binding specificity and selective antagonism of vedolizumab, an anti-alpha4beta7 integrin therapeutic antibody in development for inflammatory bowel diseases. J Pharmacol Exp Ther 2009;330:864-875.

93. Sody E, Körber A. Psoriasis induced by vedolizumab. Inflamm Bowel Dis 2017;23:E9-E11.

94. Vavricka SR, Galván JA, Dawson H, et al. Expression patterns of TNF $\alpha$, MAdCAM1, and STAT3 in intestinal and skin manifestations of inflammatory bowel disease. J Crohns Colitis 2018;12:347-354.

95. Chateau T, Bonovas S, Le Berre C, Mathieu N, Danese S, Peyrin-Biroulet L. Vedolizumab treatment in extra-intestinal manifestations in inflammatory bowel disease: a systematic review. J Crohns Colitis 2019;13:1569-1577.

96. Kavanaugh A, Puig L, Gottlieb AB, et al. Efficacy and safety of ustekinumab in psoriatic arthritis patients with peripheral arthritis and physician-reported spondylitis: post-hoc analyses from two phase III, multicentre, double-blind, placebocontrolled studies (PSUMMIT-1/PSUMMIT-2). Ann Rheum Dis 2016;75:1984-1988.

97. Greb JE, Gottlieb AB, Goldminz AM. High-dose ustekinumab for the treatment of severe, recalcitrant pyoderma gangrenosum. Dermatol Ther 2016;29:482-483.

98. Tillack C, Ehmann LM, Friedrich M, et al. Anti-TNF antibodyinduced psoriasiform skin lesions in patients with inflammatory bowel disease are characterised by interferon- $\gamma$ expressing Th1 cells and IL-17A/IL-22-expressing Th17 cells and respond to anti-IL-12/IL-23 antibody treatment. Gut 2014; 63:567-577.

99. Čarija A, Ivić I, Marasović-Krstulović D, Puizina-Ivić N. Paradoxical psoriatic arthritis in a patient with psoriasis treated with ustekinumab. Rheumatology (Oxford) 2015;54:21142116.

100. Gregoriou S, Kazakos C, Christofidou E, Kontochristopoulos G, Vakis G, Rigopoulos D. Pustular psoriasis development after initial ustekinumab administration in chronic plaque psoriasis. Eur J Dermatol 2011;21:104-105.

101. Biemans VBC, van der Meulen-de Jong AE, van der Woude CJ, et al. Ustekinumab for Crohn's disease: results of the ICC registry, a nationwide prospective observational cohort study. J Crohns Colitis 2020;14:33-45.

102. Peyrin-Biroulet L, Danese S, Louis E, et al. DOP50 Effect of upadacitinib on extra-intestinal manifestations in patients with moderate to severe Crohn's disease: data from the CELEST study. J Crohns Colitis 2019;13 Suppl 1:S057.

103. Fleischmann R, Mysler E, Hall S, et al. Efficacy and safety of tofacitinib monotherapy, tofacitinib with methotrexate, and adalimumab with methotrexate in patients with rheumatoid arthritis (ORAL Strategy): a phase 3b/4, double-blind, headto-head, randomised controlled trial. Lancet 2017;390:457468.

104. Satta R, Pes GM, Rocchi C, Pes MC, Dore MP. Is probiotic use beneficial for skin lesions in patients with inflammatory bowel disease? J Dermatolog Treat 2019;30:612-616.

105. Sanges M, Valente G, Rea M, et al. Probiotics in spondyloarthropathy associated with ulcerative colitis: a pilot study. Eur Rev Med Pharmacol Sci 2009;13:233-234.

106. Manser CN, Borovicka J, Seibold F, et al. Risk factors for complications in patients with ulcerative colitis. United European 
Gastroenterol J 2016;4:281-287.

107. Huang V, Mishra R, Thanabalan R, Nguyen GC. Patient awareness of extraintestinal manifestations of inflammatory bowel disease. J Crohns Colitis 2013;7:e318-e324.

108. Murthy SK, Nguyen GC. Venous thromboembolism in inflammatory bowel disease: an epidemiological review. Am J Gastroenterol 2011;106:713-718.

109. Kelso M, Feagins LA. Can smartphones help deliver smarter care for patients with inflammatory bowel disease? Inflamm Bowel Dis 2018;24:1453-1459.

110. Walsh A, Matini L, Hinds C, et al. Real-time data monitoring for ulcerative colitis: patient perception and qualitative analysis. Intest Res 2019;17:365-374.

111. Atreja A, Otobo E, Ramireddy K, Deorocki A. Remote patient monitoring in IBD: current state and future directions. Curr Gastroenterol Rep 2018;20:6. 